CRITICAL BEHAVIOUR

\section{Monkey business}

Nat. Commun. 8, 14301 (2017)

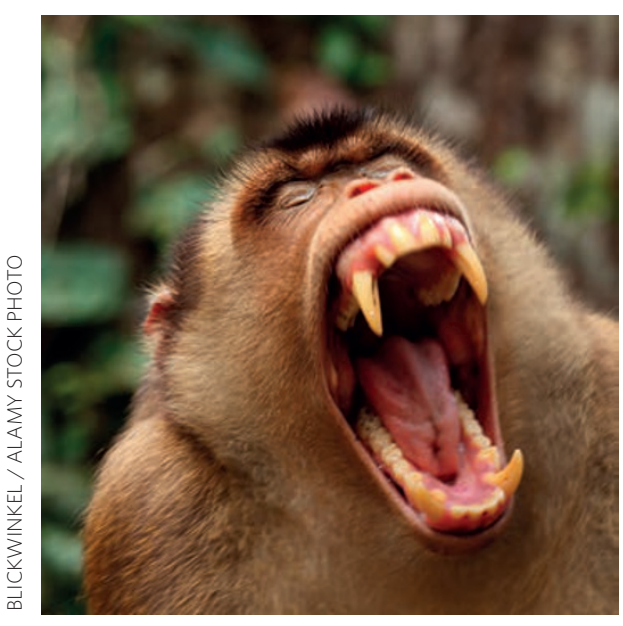

Evidence is mounting for the idea that living systems - such as flocks of birds and schools of fish - sit close to a critical point. But what if the social behaviour of monkeys was also influenced by criticality? Bryan Daniels and colleagues sought to find out, by examining the distribution of fight sizes in a population of pigtailed macaques (Macaca nemestrina; pictured). They found signs that these primates indeed hover near a tipping point, trading a certain robustness for the adaptability associated with criticality. But more surprising, perhaps, was the indication that they actively tune their distance from criticality by exploiting the group's heterogeneity.

Daniels et al. looked at fight sizes from a group of 48 monkeys, defining an operational definition of criticality for finite systems based on the Fisher information and a collective instability measure. They found that the influence the monkeys had over the collective fight-joining behaviour was heterogeneous, and that the monkeys with the greatest sway over average fight sizes were those with the most control over changes in sensitivity. How well these ideas map to us higher primates remains to be seen. AK

\section{QUANTUM CURRENCY}

\section{No safe bets}

npj Quant. Inf. 3, X (2017); doi: 10.1038/s41534017-0010-x [Full ref. to come]

Unknown quantum states cannot be perfectly copied. This is the no-cloning theorem, making quantum information an ideal medium for cryptography. By embedding a quantum token into a banknote, it may be possible to create a currency that is invulnerable to any cloning attacks. In reality, however, quantum data are always prone to external influence, and some level of noise has to be tolerated in order to harness the benefits of quantum technologies. This inevitably leads to the possibility of forgery, placing the safety of quantum money under question.

Karol Bartkiewicz and colleagues have reported an experimental realization of quantum money. Instead of focusing on a practical implementation, though, they analysed the possibility of attacking their setup by exploiting its imperfections. The authors applied optimal cloning strategies and tried to find the conditions under which quantum money is vulnerable. Their results - and the solutions they pose may cause us to rethink the security of quantum communication and quantum technologies.

\section{D MATERIALS}

\section{Amorphous and fluctuating}

Proc. Natl Acad. Sci. USA

http://doi.org/bzn6 (2017)

\title{
X-RAY BURSTS
}

\section{Blame the gap}

Neutron star binaries are known for their X-ray bursts. Most of the time these are wellbehaved, regular flashes, or type-I bursts. But there are a couple of anomalies. One is the binary system known as Rapid Burster, which also displays sudden, erratic flashes: type-II bursts. Rapid Burster was discovered in the 1970s, but the origin of its type-II bursts is still poorly understood. But now, using simultaneous observations from three space X-ray telescopes to study Rapid Burster, Jakob van den Eijnden and colleagues have likely found the culprit.

The neutron star continuously feeds on the gas from its accretion disk, burping out type-I flashes. But as van den Eijnden etal. discovered, there is a hole in the middle of Rapid Burster's accretion disk. The gap, with a radius of $87 \mathrm{~km}$, is created by the star's extremely strong magnetic field and keeps the gas at bay. So even though both the star and its disk are spinning, the gas accumulates at the inner edge. Then, when it has gained sufficient speed, the gas suddenly breaks through into the star, creating a spectacular burst of $X$-rays.

When graphene hit the condensed-matter scene in 2004, many a (theoretical) physicist got confused. Didn't the celebrated MerminWagner theorem, penned nearly 40 years earlier, implicate that $2 \mathrm{D}$ crystals cannot exist? Subtleties surround this apparent paradox, but the theorem does imply that in two dimensions, at finite temperature, long-wavelength density fluctuations lead to particle displacements that grow logarithmically with distance. In graphene's case, phonons save the day, because they mediate long-range density fluctuations.

Bernd Illing and colleagues have now contemplated Mermin-Wagner fluctuations in 2D amorphous solids. A comparison of experimental structural data from 2D and 3D colloidal crystalline and glassy systems with simulations of 2D crystals enabled the authors to make a series of noteworthy conclusions.

After confirming that the fluctuations do occur in $2 \mathrm{D}$ glasses, and hence that periodicity is not a requirement, Illing et al. showed that they provide a channel for structural decay - a situation different from the 3D case. The presence of MerminWagner fluctuations does not, however, lead to different microscopic glass transition mechanisms for $2 \mathrm{D}$ and $3 \mathrm{D}$ systems.

\section{BLACK HOLES}

\section{New horizons for magnonics \\ Phys. Rev. Lett. 118, 061301 (2017)}

Stephen Hawking's 1974 prediction that quantum effects near the event horizon enable black holes to emit thermal $Y L$ radiation - meaning that they are not completely black - could provide a fascinating experimental means for probing the intersection of general relativity and quantum mechanics. Sadly, however, the temperatures associated with Hawking radiation from astrophysical black holes are far too small to be detectable within the foreseeable future. But a proposal by Alejandro Roldán-Molina and colleagues could allow the physics of Hawking radiation to be probed inside magnetic materials.

Although astrophysical black holes may be out of experimental reach, analogues of black hole horizons can and have been created for sound waves, based on transitions between subsonic and supersonic flow. Roldán-Molina et al. showed theoretically that a similar analogy can be applied to magnetic systems, with the interactions between spin currents and magnetization dynamics giving rise to black- or white-hole horizons for magnons - the quanta of spin waves. As the predicted Hawking temperatures could be 
as large as $1 \mathrm{~K}$, magnonic black holes could provide an experimentally accessible means for probing Hawking radiation physics in the solid state.

Written by Luke Fleet, lulia Georgescu,

Abigail Klopper, Yun Li and Bart Verberck. 